BMJ Open Sport \& Exercise Medicine

\title{
Effect of high-intensity resistance exercise on cardiometabolic health in older men with osteosarcopenia: the randomised controlled Franconian Osteopenia and Sarcopenia Trial (FrOST)
}

\author{
Wolfgang Kemmler (D) ," Matthias Kohl, ${ }^{2}$ Simon von Stengel, ${ }^{1}$ Daniel Schoene ${ }^{1}$
}

To cite: Kemmler W, Kohl M, von Stengel S, et al. Effect of high-intensity resistance exercise on cardiometabolic health in older men with osteosarcopenia: the randomised controlled Franconian Osteopenia and Sarcopenia Trial (FrOST). BMJ Open Sport \& Exercise Medicine 2020;6:e000846. doi:10.1136/ bmjsem-2020-000846

Accepted 14 December 2020

\section{Check for updates}

C) Author(s) (or their employer(s)) 2020. Re-use permitted under CC BY-NC. No commercial re-use. See rights and permissions. Published by BMJ.

${ }^{1}$ Institute of Medical Physics, Friedrich-Alexander-University Erlangen-Nürnberg, Erlangen, Germany

${ }^{2}$ Faculty Medical and Life Sciences, Furtwangen University of Applied Sciences, Furtwangen, Germany

\section{Correspondence to} Professor Wolfgang Kemmler; wolfgang.kemmler@imp.unierlangen.de

\section{ABSTRACT}

Objectives Sarcopenia is related to the metabolic syndrome (MetS), a cluster of cardiometabolic risk-factors (CRF). Most exercise trials apply aerobic rather than resistance exercise to address CRF, while the strategy for maintaining muscle and bone is the opposite. However, there is considerable evidence that resistance exercise positively affects CRF. In the present study, we determined the effect of high-intensity resistance exercise training (HIT-RT) on CRF represented by the MetS in older men. Methods Forty-three osteosarcopenic and predominately obese older men ( $>72$ years) living independently in Erlangen-Nürnberg, Germany were randomly assigned to two study arms. The HIT-RT group $(\mathrm{n}=21)$ conducted a periodised high intensity/effort protocol dedicated to muscle and bone mass and function two times per week, while the control group (CG: $n=22$ ) maintained their habitual physical activities. Both groups were supplemented with protein, cholecalciferol and calcium. Study outcomes presented here were the MetS-Z (MetSZ) score and its underlying risk-factors.

Results After 18 months of intervention, we observed significant effects for the MetSZ score $(p<0.001)$, with significant improvements in the HIT-RT and significant worsening in the CG. In detail, all parameters constituting the MetS contributed to this result, however, only waistcircumference, $\mathrm{HDL}$-cholesterol and mean arterial blood pressure revealed significant effects. No adverse events were reported and high adherence was determined for the HIT-RTprotocol.

Conclusion Continuously supervised HIT-RT is an effective, attractive, feasible and safe method to address cardiometabolic risk factors in community-dwelling men aged 72 years and older. Further, considering the proven effects on musculoskeletal risk factors, the present results indicate a more prominent role for HIT-RT within nonpharmacological prevention strategies of older adults. Trial registration number NCT03453463.

\section{INTRODUCTION}

Sarcopenia characterises the increased agerelated and/or immobility-related reduction of muscle mass and function. Apart from

\section{Key messages}

High-intensity resistance exercise training (HIT-RT) is highly effective for addressing the metabolic syndrome in older men with low muscle and bone mass.

- Consistently supervised and carefully guided HIT-RT is safe, feasible and attractive for older men with low affinity to resistance exercise so far.

- Due to the multimorbidity aspect of many older people, HIT-RT should be assigned a more prominent role within the prevention and therapy of chronic diseases.

functional aspects, skeletal muscles are major sites for glucose uptake and deposition. ${ }^{1}$ There is considerable evidence that sarcopenia increases insulin resistance, thereby progressively inducing diabetes and metabolic syndrome (MetS). ${ }^{2}$ Thus, considering the cardiometabolic relevance of muscle, interventions that can counteract sarcopenia are of high priority. The most effective sarcopenia therapy might be (dynamic) resistance exercise (DRT) ${ }^{3}$ However, as the majority of older people show limited affinity to resistance exercise, ${ }^{4}$ time effective, consistently supervised DRT supported by dietary supplements could be a feasible option for this cohort.

High-intensity resistance exercise training (HIT-RT) defined as single set resistance exercise conducted with high effort ${ }^{5}$ could be such a time-efficient vehicle. In a recent study, we compared the effect of a time-efficient HIT-RT protocol with a multi-set RT protocol in predominately overweight men aged 30-50 years. ${ }^{6-8}$ Apart from slightly (non-significant) higher effects on cardiometabolic risk factors, ${ }^{6}{ }^{7}$ HIT-RT demonstrates comparable effects on lean body mass (LBM); however, only in combination with protein supplementation. $^{8}$ Thus, while exercise volume of the 
RT might not be the critical aspect, there is considerable evidence, although not undisputed, that high exercise intensity is more favourable for affecting cardiometabolic parameters compared with low or moderate intensity exercise. $^{9-11}$ Further, in view of the low effort needed to cover the increased protein demand in older age ${ }^{12}$ with protein supplements, the combination of supervised HIT-RT and customised protein supplementation might be a promising approach for this vulnerable cohort.

The aim of the present contribution was thus to determine the effect of a state-of-the-art HIT-RT combined with moderately dosed (whey) protein supplementation on the MetS as a cluster of cardiometabolic risk factors ${ }^{13}$ in community dwelling older men with osteosarcopenia. Our hypothesis was that the MetSZ (MetSZ) score improves significantly in the HIT-RT group compared with the non-training control group (CG). Our secondary hypotheses were (i) mean arterial blood pressure (MAP) and (ii) waist circumference decrease significantly, while (iii) resting glucose, (iv) triglycerides and (v) HDL-cholesterol (HDL-C) do not change significantly compared with control.

\section{MATERIAL AND METHODS Study design}

The Franconian Osteopeniaand Sarcopenia Trial (FrOST) is a randomised controlled exercise trial with a balanced parallel group design that included community-dwelling $(\mathrm{CDW})$ older men with osteosarcopenia. ${ }^{14}$ Primary study outcomes of FrOST were skeletal muscle mass and bone mineral density ${ }^{15}$; the present publication focuses on changes of the MetS, as determined from baseline (June 2018) to study end (December 2019).

\section{Patient and public involvement}

Patients and/or the public were not materially involved in the study design, conduction or dissemination of this research.

\section{Participants}

Participant recruitment and flow were reported in detail in preceding publications, ${ }^{16}{ }^{17}$ thus only a condensed summary will be provided. Briefly, 242 CDW men 72 years and older, participants of a preceding epidemiological study ${ }^{18}$ were invited to a follow-up assessment. One hundred and eighty men responded ${ }^{19}$ and were assessed for eligibility (figure 1). Applying the exclusion criteria (i) secondary osteoporosis, (ii) history of hip fracture, (iii) resistance type exercise $>45 \mathrm{~min} /$ week, (iv) amputations of limbs or cardiac pacemaker, (v) implementation of glucocorticoid therapy $>7.5 \mathrm{mg}$ /day during the last 2 years, (vi) cognitive impairments, (vii) alcohol consumption $>60 \mathrm{~g}$ ethanol per day, (viii) skeletal muscle mass index $>7.50 \mathrm{~kg} / \mathrm{m}^{2}$ as determined by bio-impedanceanalysis, left 103 men preliminary eligible. Applying further dual energy X-ray absorptiometry (DXA), men were included in FrOST when featuring (i) sarcopenia $\left(<7.26 \mathrm{~kg} / \mathrm{m}^{220}{ }^{21}\right)$ and (ii) osteopenia. ${ }^{22}$ Finally, $43 \mathrm{men}$

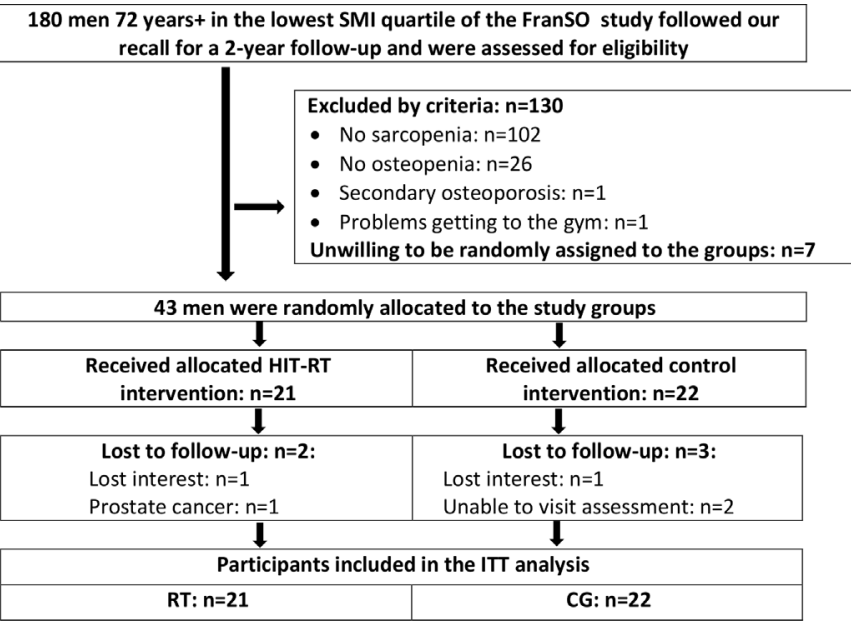

Figure 1 Participant flow through the study. CG, control group; FranSO: Franconian Sarcopenic Obesity trial; HIT-RT, high-intensity resistance exercise training; ITT, intention-totreat; SMI: skeletal muscle mass index.

were willing to be randomly allocated either to an exercise (HIT-RT: $n=21)$ or CG $(n=22)$ and were included in FrOST. Figure 1 shows participant flow through the study.

\section{Randomisation procedures}

Participants randomly allocated themselves to the study groups by drawing lots. Lots were put in small opaque plastic containers and placed in a bowl by a person not involved in the present project. Neither participants nor researchers knew the group allocation before the lots were drawn and the capsules were opened (allocation concealment). After randomisation, the primary investigator (WK) enrolled participants and instructed them in detail about their study status and corresponding do's and don'ts (see below).

\section{Blinding}

Our blinding strategy focused on outcome assessors and test assistants who were unaware of the participants' group status (HIT-RT or CG) and were not allowed to ask, either.

\section{Study procedure, intervention}

Dietary supplementation

All participants were provided with cholecalciferol, calcium and whey protein supplements in order to reach current recommendations. ${ }^{12}{ }^{23}$ In line with the Prot-Age research group ${ }^{12}$ we intended a total whey protein (Active PRO80, inkospor, Roth, Germany) intake of $1.5 \mathrm{~g} / \mathrm{kg}$ in the HIT-RT and an intake of $1.2 \mathrm{~g} / \mathrm{kg}$ body mass/day in the CG. The chemical score of the protein product is 159 , $100 \mathrm{~g} /$ day represented a caloric value of $360 \mathrm{kcal}$ and contained $80 \mathrm{~g}$ of (whey) protein with a high L-Leucine $(9 \mathrm{~g})$ and essential amino acid (57g) component. Three hundred milligram of calcium were contained in a $25 \mathrm{~g} /$ portion of the protein powder. We recommended participants with low calcium intake to mix the protein powder 
with low fat milk $(10 \mathrm{~g} / 150 \mathrm{~mL})$ and to split doses higher than $30 \mathrm{~g} /$ day.

Based on blood analysis of $25 \mathrm{OH}$ Vitamin-D $3(25(\mathrm{OH})$ D) all participants of the HIT-RT or CG were provided with cholecalciferol (MYVITAMINS, Manchester, UK). Participants with serum concentrations below $30 \mathrm{ng} / \mathrm{mL}$ were supplemented with $10000 \mathrm{IE} /$ week (4 units $\times 2500$ IE). Participants with serum levels between 30 and $40 \mathrm{ng} /$ $\mathrm{mL}$ were requested to take two capsules of $2500 \mathrm{IE}$, that is, $5000 \mathrm{IE} /$ week.

Based on the dietary calcium questionnaire (Rheumaliga, Switzerland) we aimed to ensure a calcium intake of $\approx 1000 \mathrm{mg} /$ day in all the participants. The required additional daily calcium was provided by calcium capsules (Sankt Bernhard, Bad Dietzenbach, Germany).

\section{Intervention: resistance exercise}

The FrOST study intervention was described in detail in earlier publications, ${ }^{15} 17$ thus only a brief summary of the intervention will be provided. Of importance, the exercise intervention of the present study focused exclusively on isolated dynamic resistance training on strength devices (MEDX, Ocala, Florida, USA). Twelve to fourteen exercises selected from a pool of 18 exercises (calf raises, leg press, -extension, leg curls, -adduction, -abduction, hip extension, latissimus front pulleys, pull-overs, seated rowing, back extension, inverse fly, bench press, military press, lateral raises, butterfly with extended arms, crunches, lateral crunches) were prescribed per session. The exercise protocol scheduled two sessions of 35-50 min/week, consistently supervised by licensed trainers. Of note, on average one trainer supervised no more than six participants. Men in the HIT-RT were provided with detailed training records that prescribed exercises, number of sets, number of repetitions (reps), movement velocity and (absolute) exercise intensity. The latter was specified by prescribing a range of reps (ie, 7-9 reps) and the set endpoint. ${ }^{24}$ Exercise protocols of each training phase were introduced beforehand in joint meetings. After a briefing and conditioning phase of 12 weeks, we introduced the single set HIT-RT structured in linearly periodised mesocycles with 2-3 blocks of 4 weeks with each fourth week as an active regeneration week. Relative intensity during the 4-week blocks averaged between $60 \% 1$ repetition maximum (RM) for week 1 and up to $85 \% 1$ RM for week 3. During the following mesocycles, we consistently manipulated movement velocity, breaks between set/exercises and introduced explosive movements during the concentric phase. We also switched from a non-RM (nRM) ${ }^{25}$ to the RM (Set endpoint when trainees properly complete the final repetition but if the next repetition were attempted they would definitely achieve momentary failure. ${ }^{24}$ However, according to the definition, sets conducted with high velocity were terminated when explosive velocity was no longer possible.) approach. ${ }^{24}$ We introduced the superset approach during the fourth and the drop-set approach during the fifth mesocycle. After 1 year (ie, mesocycle
6 ), we tested the momentary failure approach (Briefly 'inability to realise the concentric phase of the current rep'.), however after 2-3 weeks of training we decided together with the participants to abstain from this very exhaustive training method. Apart from slight alterations, no further relevant changes of the training protocol were made during the last 5 months of the 18-month intervention. Taken together, our exercise programme provided a time-efficient periodised DRT for most minor and all major muscle groups with high intensity (up to $85 \%$ 1RM), high effort (RM, intensifying strategies) and high movement velocity and introduced regular active rest periods (60\% 1RM, nRM).

Attendance and compliance with the exercise protocol were checked by instructors and by the gym's chip card system. Compliance with the dietary supplementation was monitored by the distribution records, the research assistants' regular phone calls and personal interviews at follow-ups.

Of importance, all participants were requested not to change their physical activity and exercise habits outside the present study intervention. Further, all the men were asked to maintain their dietary habits.

\section{Study outcomes}

Main outcome of the present study

- MetsZ score ${ }^{26}$ based on the International Diabetes Federation (IDF) criteria of the MetS ${ }^{13}$ as assessed at baseline and at 18-month follow-up.

Secondary outcome of the present study

- Parameters constituting the MetS according to IDF $^{13}$ as assessed at baseline and at 18-month follow-up.

- Waist circumference.

- MAP.

- Glucose.

- Triglycerides.

- HDL-cholesterol.

\section{Changes of trial outcomes after trial commencement}

Due to a technical defect, DXA assessments were not conducted at the 6-month follow-up as intended.

\section{Assessments}

Participants were asked to restrain from intense physical activity and exercise 48 hours preassessment. Baseline and 18-month follow-up tests were conducted by the same researcher at the same time of day.

\section{Outcome parameters}

Height was measured using a stadiometer and body mass was determined via direct-segmental, multi-frequency Bio-Impedance-Analysis (DSM-BIA; InBody 770, Seoul, Korea). Waist circumference was determined as the minimum circumference between the distal end of the rib cage and the top of the iliac crest along the midaxillary line.

After an overnight fast, blood was drawn in a sitting position from an antecubital vein between 07:00 and 
09:00. Serum samples were centrifuged for $20 \mathrm{~min}$ at 3000 rpm and analysed by the 'Zentrallabor' of the Medical Department, FAU. Glucose, total cholesterol, HDL and LDL-cholesterol and triglycerides (Olympus Diagnostica GmbH, Hamburg, Germany) were determined.

Immediately after $10 \mathrm{~min}$ of rest, blood pressure (RR) was determined in a lying position with an automatic oscillometric device (Bosco, Bosch, Jungingen, Germany). Subjects were requested to arrive in a relaxed, non-fasting condition but having refrained from coffee or tea for at least 2 hours prior to testing. MAP was calculated (diastolic RR+diastolic RR+systolic RR)/3.

In order to generate a continuous score for MetS, we calculated the MetSZ score based on the approach of Johnson et $a l^{26}{ }^{26}$ However, unlike Johnson et $a l,{ }^{26}$ we applied the most recent MetS definition, that is, that of the International Diabetes Federation. ${ }^{13}$ In detail, the Z-score was calculated using: [(40-HDL-cholesterol) / SD HDL-C $]+[($ triglycerides -150$) / \mathrm{SD}$ TriGly $]+[(\mathrm{Glu}-$ cose-100)/SD Glucose $]+[$ (waist circumference-88)/SD $\mathrm{WC}]+[(\mathrm{MAP}-100) / \mathrm{SD} \mathrm{MAP}]$. Of importance for interpreting the results, negative Z-scores and negative Z-score changes can be considered as favourable.

Baseline characteristics and confounding factors (ie, lifestyle, diseases, medication, physical activity) were assessed at baseline and follow-up by standardised questionnaires $^{1627}$ and personal interviews. Particular emphasis was placed on monitoring changes of parameters with potential confounding effects on our results. This included physical activity and exercise habits, diet, diseases and medication.

The participants' dietary intake was assessed at baseline, 6, 12 and 18 months by a 4-day dietary protocol kept by all participants. The consumed food was analysed using the Freiburger Ernährungs-Protokoll (Freiburger Nutrition Protocol) (nutri-science, Hausach, Germany).

\section{Sample size}

The sample size calculation for the FrOST project was based on integral bone mineral density (BMD) at the lumbar spine as determined by Quantitative Computed Tomography (QCT), an outcome not addressed in this contribution.

\section{Statistical analysis}

Intention-to-treat (ITT) analysis was applied and included all participants randomly assigned to the HIT-RT or CG. Multiple imputation (ITT) was calculated using $\mathrm{R}$ statistics software ( $\mathrm{R}$ Development Core Team Vienna, Austria) in combination with Amelia II. ${ }^{28}$ Imputation was repeated 100 times and worked well in all cases as confirmed by overimputation diagnostic plots ('observed vs imputed values'). Normal distribution of the data was checked by statistical (Shapiro-Wilks) and graphical (qq-plots) tests. Based on the corresponding results, study outcomes addressed here were analysed by dependent t-tests for within group changes. Pairwise t-test comparisons (HIT-RT vs CG) for intragroup changes with
Table 1 Baseline characteristics of the CG and HIT-RT groups

\begin{tabular}{|c|c|c|c|}
\hline Variable & $\begin{array}{l}\text { CG } \\
(n=22)\end{array}$ & $\begin{array}{l}\text { HIT-RT } \\
(n=21)\end{array}$ & $P$ value \\
\hline Age (years) & $79.2 \pm 4.7$ & $77.8 \pm 3.6$ & 0.262 \\
\hline Body mass index $\left(\mathrm{kg} / \mathrm{m}^{2}\right)^{\star}$ & $24.5 \pm 1.9$ & $25.0 \pm 3.0$ & 0.515 \\
\hline Body fat rate (DXA) $(\%) \dagger$ & $34.5 \pm 6.1$ & $33.6 \pm 4.0$ & 0.563 \\
\hline LBM $\left(\mathrm{kg} / \mathrm{m}^{2}\right) \dagger$ & $6.89 \pm 0.31$ & $7.01 \pm 0.27$ & 0.671 \\
\hline MetS-prevalence (n) & 7 & 8 & 0.666 \\
\hline Diabetes mellitus type II (n) & 1 & 1 & 0.960 \\
\hline Hypertension (n)‡ & 7 & 11 & 0.172 \\
\hline Physical activity (index)§ & $4.15 \pm 1.53$ & $4.45 \pm 1.32$ & 0.490 \\
\hline Regular exercise $\geq 1 \times$ week $(n)$ & 5 & 5 & 0.931 \\
\hline ๆALEF, LLFDI (index)ף & $1.73 \pm 0.82$ & $1.87 \pm 1.05$ & 0.646 \\
\hline $25(\mathrm{OH}) \mathrm{D}(\mathrm{nmol} / \mathrm{L})^{* *}$ & $54.0 \pm 21.1$ & $43.8 \pm 17.5$ & 0.126 \\
\hline Energy intake (MJ/day)†† & $9.39 \pm 2.42$ & $8.84 \pm 1.71$ & 0.407 \\
\hline Smokers (n) & 4 & 3 & 0.959 \\
\hline
\end{tabular}

*Via bio impedance analysis.

†Via dual-energy X-ray absorptiometry (DXA, hological QDR 4500a, USA).

$\ddagger$ Mean arterial blood pressure>106.7RR.

$\S$ Scale from (1) very low to (7) very high. ${ }^{51}$

ף'Advanced lower extremity function', late life function disability instrument ${ }^{52}$ : (scale from (1) 'no problem' to (5) 'impossible').

${ }^{* *}$ Serum concentrations of $25(\mathrm{OH}) \mathrm{D}$ were measured

using a Roche Modular E170 Analyzer and an electro-

chemiluminescence immunoassay (ECLIA; Roche Diagnostics, Penzberg, Germany).

$\dagger \dagger$ As determined by a 4-day dietary record.

CG, control group; DXA, dual energy X-ray absorptiometry; HIT$\mathrm{RT}$, high-intensity resistance exercise training; LBM, Lean Body Mass; MetS, metabolic syndrome.

pooled SD were applied to identify group differences ('effects'). In order to adjust for baseline differences, we additionally analysed the data using an analysis of covariance (ANCOVA). Standardised mean difference (SMD) according to Cohen $\left(\mathrm{d}^{29}\right)$ was calculated to determine effect sizes. We applied two-tailed tests, significance was accepted at $\mathrm{p}<0.05$.

\section{RESULTS}

\section{Baseline characteristics}

Table 1 shows participant characteristics at baseline. Most baseline characteristics were similarly distributed between the groups. However, some particularities should be noted. Accepting that a body fat rate of $27 \%-30 \%$ constitutes obesity in men, ${ }^{30}$ most participants $(75 \%-94 \%)$ can be considered as 'osteosarcopenic obese'. ${ }^{31}$ Further, serum $25(\mathrm{OH}) \mathrm{D}$ was very low in both groups.

\section{Intervention characteristics}

Altogether five participants were lost to follow-up (figure 1). No participant of the HIT-RT group quit the study for intervention-related reasons. Apart from one participant who reported temporary worsening of existing knee and shoulder pain, no resistance-training-induced 
Table 2 Baseline data and changes of the MetS-Z score in the CG and HIT-RT along with corresponding between group differences

\begin{tabular}{|c|c|c|c|c|}
\hline & $\begin{array}{l}C G \\
M V \pm S D\end{array}$ & $\begin{array}{l}\text { HIT-RT } \\
\text { MV } \pm S D\end{array}$ & $\begin{array}{l}\text { Difference } \\
\text { MV (95\% Cl) }\end{array}$ & $P$ value \\
\hline \multicolumn{5}{|c|}{ MetS-Z score (Z) } \\
\hline Baseline & $-4.22 \pm 3.70$ & $-3.14 \pm 4.66$ & - & 0.322 \\
\hline Changes & $0.80 \pm 1.71^{*}$ & $-0.88 \pm 1.33^{*}$ & $1.68(0.73$ to 2.65$)$ & 0.001 \\
\hline
\end{tabular}

${ }^{*} \mathrm{p}<0.05$.

CG, control group; HIT-RT, high-intensity resistance exercise training; MetS, metabolic syndrome; MV, mean value; SD, standard deviation.

complaints or unintended side effects were recorded. Average training attendance (rate) was $95 \% \pm 5 \%(84 \%-$ $100 \%)$ and average time expended for an exercise session varied between 35 and $50 \mathrm{~min}$. Based on distribution logs, biweekly phone calls and personal interviews we consider compliance with protein, calcium and cholecalciferol supplementation as high $(>90 \%)$.

\section{Study endpoint}

Table 2 displays study results on the MetSZ score. In summary, the MetSZ improved significantly in the HIT-RT $(p=0.012)$ and worsened significantly $(p=0.021)$ in the CG. Differences between the exercise and CGs (ie, 'effect'), both of which were supplemented with protein, cholecalciferol and calcium, were significant $(p=0.001 ; S M D=1.10)$. Applying ANCOVA to adjust for baseline differences (table 2 ) the effect remained statistically significant $(\mathrm{p}=0.001$; mean group difference: 1.47 ; $95 \%$ CI 0.56 to 2.37 ).

In detail, all parameters included in the MetS according to IDF ${ }^{13}$ contributed to the positive result on MetSZ. Waist circumference, MAP and HDL-C show significant effects (table 3), while group difference for 18-month changes of resting glucose (HIT-RT: $6.0 \pm 13.6$ vs CG: $7.9 \pm 16.5 \mathrm{mg}$ / $\mathrm{dL} ; \mathrm{p}=0.673$ ) and triglycerides (HIT-RT: $-14.2 \pm 40.2$ vs CG: $-5.7 \pm 46.9 \mathrm{mg} / \mathrm{dL} ; \mathrm{p}=0.526$ ) were non-significant. Effect sizes for group differences of waist circumference (SMD: 1.37), MAP (SMD: 0.98) and HDL-C (SMD: 0.57) average from moderate to high. Applying ANCOVA, group differences remained statistically significant for waist circumference $(\mathrm{p}=0.001$; mean group difference: $3.25 \mathrm{~cm} ; 95 \%$ CI 2.06 to $4.44 \mathrm{~cm}$ ), MAP ( $\mathrm{p}=0.013 ; 4.07 \mathrm{~mm}$ $\mathrm{Hg}$; $95 \%$ CI 0.92 to $7.22 \mathrm{~mm} \mathrm{Hg})$ and HDL-C ( $\mathrm{p}=0.042$; $4.94 \mathrm{mg} / \mathrm{dL} ; 95 \%$ CI 0.2 to $9.7 \mathrm{mg} / \mathrm{dL}$ ). In parallel, group differences of absolute changes remained non-significant for resting glucose $(\mathrm{p}=0.809)$ and triglycerides $(\mathrm{p}=0.501)$.

\section{Potentially confounding parameters}

Serum 25 $(\mathrm{OH}) \mathrm{D}$ increased similarly $(\mathrm{p}<0.001)$ in both groups. As determined by dedicated questionnaires ${ }^{27}$ and confirmed by personal interviews, participants reported no relevant changes of lifestyle, physical activity and exercise outside the HIT-RT protocol. In parallel, dietary records conducted at baseline and then after 6, 12 and 18 months did not indicate relevant changes of dietary habits. No participant reported relevant changes of pharmaceutic therapies. However, apart from the man who had to stop exercising due to prostate cancer, two men of the HIT-RT and three men of the CG reported longer periods (3-8 weeks) of physical inactivity due to diseases and hospitalisation.

\section{DISCUSSION}

The primary aim of FrOST was to determine the effects of HIT-RT on bone and muscle mass, but also its safety and feasibility in older, CDW men with osteosarcopenia and, at least in the majority (table 1), obesity. The present

Table 3 Baseline data and changes of selected parameters constituting the metabolic syndrome according to IDF ${ }^{13}$

\begin{tabular}{|c|c|c|c|c|}
\hline & $\begin{array}{l}\mathrm{CG} \\
\mathrm{MV} \pm \mathrm{SD}\end{array}$ & $\begin{array}{l}\text { HIT-RT } \\
M V \pm S D\end{array}$ & $\begin{array}{l}\text { Difference } \\
\text { MV (95\% Cl) }\end{array}$ & $P$ value \\
\hline \multicolumn{5}{|c|}{ Waist circumference (cm) } \\
\hline Baseline & $89.2 \pm 8.9$ & $92.4 \pm 11.1$ & - & 0.306 \\
\hline Changes & $1.12 \pm 2.16^{*}$ & $-1.85 \pm 2.17^{\star \star \star}$ & 2.97 (1.59 to 4.36$)$ & $<0.001$ \\
\hline \multicolumn{5}{|c|}{ Mean arterial blood pressure (MAP) (mm Hg) } \\
\hline Baseline & $99.1 \pm 8.6$ & $103.0 \pm 6.8$ & - & 0.114 \\
\hline Changes & $4.05 \pm 5.87^{\star *}$ & $-1.73 \pm 5.95$ & $5.78(2.08$ to 9.47$)$ & 0.003 \\
\hline \multicolumn{5}{|c|}{ HDL-cholesterol (HDL-C) (mg/dL) } \\
\hline Baseline & $60.2 \pm 14.0$ & $54.4 \pm 12.3$ & - & 0.165 \\
\hline Changes & $0.25 \pm 11.1$ & $6.09 \pm 9.40^{*}$ & 6.09 (0.01 to 11.67$)$ & 0.049 \\
\hline
\end{tabular}

${ }^{*} \mathrm{p}<0.05 ;{ }^{* *} \mathrm{p}<0.01 ;{ }^{* \star *} \mathrm{p}<0.001$.

$\mathrm{CG}$, control group; HIT-RT, high-intensity resistance exercise training; MV, mean value; SD, standard deviation. 
contribution focuses on the study endpoint 'MetS' as a cardiometabolic risk cluster.

\section{What does the study add?}

In summary, after 18 months of high intensity/high effort/high velocity single-set DRT, we observed a pronounced effect on the MetSZ, without relevant adverse effects. In terms of drop-out and exercise attendance, the HIT-RT protocol applied can be considered not only as (time-) effective and safe, but also as an attractive exercise method for older men with limited affinity to exercise (table 1).

In order to generate a continuous and summarised score for the MetS, we applied the Z-score approach of Johnson $e t a l,{ }^{26}$ however, we used the more recent IDF criteria ${ }^{13}$ for the MetS. A review of the literature revealed that only few exercise studies address the MetSZ, and even fewer focus on resistance-type exercise ${ }^{62-35}$ In summary, Bateman $e t a l^{32}$ and Earnest $e t a l^{33}$ did not report positive effects of hypertrophy-orientated multiple set DRT 3 days per week for $4^{32}$ or 9 months ${ }^{33}$ with 73 people with type 2 diabetes, $30-75$ years old ${ }^{33}$ or 66 overweight, sedentary people $18-70$ years old. ${ }^{32}$ While one study ${ }^{35}$ focused on whole-body electromyostimulation and will be not discussed here (... the study demonstrated significant effects on the MetSZ-Score in older cdw women with sarcopenic obesity), two other studies applied a comparable HIT-RT protocol with ${ }^{34}$ or without protein supplementation $^{634}$ over 4 months, although with 23 or 40 predominately overweight/obese men 30-50 years old. Both studies reported significant positive effects on the MetSZ (SMD: $1.08^{34}$ ). Additional protein supplementation did not significantly increase the effect, a finding that is supported by other studies. ${ }^{36}{ }^{37}$ Apart from participant characteristics, the main differences to the studies of Bateman $e t a l^{32}$ and Earnest $e t a l^{33}$ is the RT-exercise protocol.

While the latter studies applied an extensive multiple set with moderate exercise intensity and effort, we focus on a time-effective, although more strenuous, intervention with high effort (RM) and intensity (up to 85\% 1RM) combined with periods of explosive movements. Indeed, there is some evidence that parameters constituting the MetS are sensitive to exercise intensity $\left(\mathrm{eg},{ }^{9-11}\right)$, although this is not undisputed $\left(\mathrm{eg},{ }^{38}\right)$. Due to our study design, we are unable to contribute to this important issue. However, our rationale for applying HIT-RT is its time efficiency. The present study indicates that $2 \times \approx 45 \mathrm{~min} /$ week are sufficient to improve both relevant cardiometabolic and also musculoskeletal parameters ${ }^{15}$ in this cohort of older men with osteosarcopenia, living independently in the community. However, there is an ongoing discussion whether HIT-RT, ${ }^{5}$ with high intensity/effort/velocity protocols can be considered suitable for older cohorts. While high velocity during resistance exercise is reported to be fully suitable and tolerable for the older subject, ${ }^{3940}$ the high effort aspect ${ }^{41}$ of HIT-RT might be viewed critically $\left(\right.$ review $i^{42}$ ) for older people. We feel that some concessions will be necessary to implement effective, safe and attractive HIT-RT protocols, namely: (1) careful conditioning and briefing period; (2) continuous supervision by certified trainers; (3) periodised protocols with varying relative (\%1RM) and absolute intensity ('effort') combined with (4) regular 'active regeneration' phases; (5) reasonable mix of $\mathrm{nRM}$ and $\mathrm{RM}$ sets within the session; (6) explosive movements not conducted at RM and (7) detailed briefing and introduction of the 'new' training logs in regular meetings. After a testing period of 2-3 weeks, we (participants and researcher) opted to drop the 'momentary failure approach', ${ }^{24}$ thus our DRT might not be a 'purebred' HIT-RT protocol. Obviously, 'work to failure' can be considered as a red line for this older cohort, at least in our study.

\section{Study limitations}

Some limitations and features may aggravate a simple interpretation of our results. (1) We applied a combined effect of HIT-RT and dietary supplementation, thus we are unable to attribute the cardiometabolic effects completely to HIT-RT. However, with respect to whey protein supplementation differing between the groups $(1.2 \mathrm{~g} / \mathrm{kg} /$ day vs $1.5 \mathrm{~g} / \mathrm{kg} /$ day), there is no conclusive evidence for positive effects on MetS indices. ${ }^{43-45}$ (2) The METS-Z score might not be the most frequently used cardiometabolic outcome. However, the continuous scale of this cluster allows a precise, combined analysis of risk factors without generating multiplicity. (3) Monitoring training logs and participant compliance with exercise intensity prescriptions, ${ }^{17}$ we estimate that $25 \%-30 \%$ of the RM sets were conducted with too low effort. (4) One may challenge the concept of easy feasibility of a supervised gym-based in general. Although home-based exercise programmes might be indeed more feasible for older people, we feel that HIT-RT protocols in previously untrained older men have to be conducted in a supervised setting using dedicated resistance devices (MEDX) particularly for reasons of safety and compliance. ${ }^{46}$ Accepting this approach, we think that $2 \times 35-50$ min closely supervised HIT-RT per week on easy-to-use resistance machines is feasible. (5) The sample size calculation of FrOST was based on a musculoskeletal outcome (BMD), and thus from a dedicated methodological point of view, one might consider it inadequate for addressing the present research topic. However, considering the initial sample site $(n=21)$ along with the ITT/imputation strategy, the continuous score of the MetSZ score and the promising exercise programme that demonstrate significant effects in, although, younger cohorts, ${ }^{9}{ }^{11}$ our approach to address the MetSZ score in this cohort of older men with osteosarcopenia and predominately overweight/obesity should be considered reasonable.

\section{CONCLUSION}

It is debatable whether our results can be simply generalised to healthy older cohorts. However, some aspects of sarcopenia, such as inflammation, ${ }^{47}$ mitochondrial 
abnormalities, ${ }^{48}$ oxidative stress ${ }^{49}$ are factors that tend to decrease $\mathrm{e}^{50}$ the effects generated by HIT-RT (and dietary supplements), and hence results should be at least comparably high in healthy older men. However, we conclude that HIT-RT is a highly effective, safe, feasible and attractive training method to fight cardiometabolic risk particularly in predominately overweight-obese older men.

Acknowledgements The authors are very grateful for the support of Kieser Training (Erlangen, Germany), which provided the gym and exercise supervision support. The authors further acknowledge the contribution of the Institute of Radiology, FAU.

Contributors WK, MK, SvS and DS designed the study, carried out data analysis and/or interpretation, drafted the manuscript and revised the manuscript. WK accepts responsibility for the integrity of the data sampling, analysis and interpretation.

Funding The study was funded by the Institute of Medical Physics (IMP), FriedrichAlexander University of Erlangen-Nürnberg, with support from the non-profit organizationorganisation "Netzwerk Knochengesundheit e.V.", a local health sport club closely associated with the IMP, FAU.

Competing interests None declared.

Patient consent for publication Not required.

Ethics approval The project was planned and initiated by the Institute of Medical Physics, University of Erlangen-Nürnberg (FAU), Germany. The FAU University Ethics Committee (number 67_15b and 4464b) and the Federal Bureau of Radiation Protection (BfS, number Z 5-2246212-2017-002) approved the trial. The study fully complies with the Declaration of Helsinki 'Ethical Principles for Medical Research Involving Human Subjects'. All study participants gave their written informed consent after receiving detailed information.

Provenance and peer review Not commissioned; externally peer reviewed.

Data availability statement Data are available upon reasonable request. The anonymised data used to support the findings of this study are available from the corresponding author upon request.

Open access This is an open access article distributed in accordance with the Creative Commons Attribution Non Commercial (CC BY-NC 4.0) license, which permits others to distribute, remix, adapt, build upon this work non-commercially, and license their derivative works on different terms, provided the original work is properly cited, appropriate credit is given, any changes made indicated, and the use is non-commercial. See: http://creativecommons.org/licenses/by-nc/4.0/.

ORCID iD

Wolfgang Kemmler http://orcid.org/0000-0003-3515-0669

\section{REFERENCES}

1 Klip A, Pâquet MR. Glucose transport and glucose transporters in muscle and their metabolic regulation. Diabetes Care 1990;13:228-43.

2 Reaven GM, lecture B. Role of insulin resistance in human disease. Diabetes 1988;37:1595-607.

3 Dent E, Morley JE, Cruz-Jentoft AJ, et al. International clinical practice guidelines for sarcopenia (ICFSR): screening, diagnosis and management. J Nutr Health Aging 2018;22:1148-61.

4 Loustalot F, Carlson SA, Kruger J, et al. Muscle-strengthening activities and participation among adults in the United States. Res $Q$ Exerc Sport 2013;84:30-8.

5 Gießing J. HIT-Hochintensitätstraining Novagenics-Verlag: Arnsberg, 2008.

6 Kemmler W, Kohl M, Stengel Svon. Effects of high intensity resistance training versus whole-body Electromyostimulation on cardiometabolic risk factors in untrained middle aged males. A randomized controlled trial. J Sports Res 2016;3:44-55.

7 Kemmler W MT, von Stengel S, Bebenek M. Effekte unterschiedlicher Krafttrainingsprogramme auf das metabolische syndrom die PUSH-Studie. Dtsch Z Sportmed 2014;63:191.

8 Wittke A, von Stengel S, Hettchen M, et al. Protein supplementation to augment the effects of high intensity resistance training in untrained middle-aged males: the randomized controlled push trial. Biomed Res Int 2017;2017:3619398.

9 Kemmler W, Lell M, Scharf M. Hoch- versus moderat-intensive Laufbelastung - Einfluss auf kardiometabolische Risikogrößen bei untrainierten Männern. Die randomisierte, kontrollierte RUSH-Studie. DMW 2015;140:7-13.

10 Swain DP, Franklin BA. Comparison of cardioprotective benefits of vigorous versus moderate intensity aerobic exercise. Am J Cardiol 2006;97:141-7.

11 Sheikholeslami Vatani D, Ahmadi S, Ahmadi Dehrashid K, et al. Changes in cardiovascular risk factors and inflammatory markers of young, healthy, men after six weeks of moderate or high intensity resistance training. J Sports Med Phys Fitness 2011;51:695-700.

12 Bauer J, Biolo G, Cederholm T, et al. Evidence-based recommendations for optimal dietary protein intake in older people: a position paper from the PROT-AGE Study Group. J Am Med Dir Assoc 2013:14:542-59.

13 Alberti KGMM, Zimmet P, Shaw J. Metabolic syndrome--a new world-wide definition. A Consensus Statement from the International Diabetes Federation. Diabet Med 2006;23:469-80.

14 World Medical Association. World Medical association Declaration of Helsinki: ethical principles for medical research involving human subjects. JAMA 2013;310:2191-4.

15 Kemmler W, Kohl M, Fröhlich M, et al. Effects of high-intensity resistance training on osteopenia and sarcopenia parameters in older men with Osteosarcopenia-One-Year results of the randomized controlled Franconian osteopenia and sarcopenia trial (frost). J Bone Miner Res 2020;35:1634-44

16 Lichtenberg T, von Stengel S, Sieber C, et al. The favorable effects of a high-intensity resistance training on sarcopenia in older community-dwelling men with osteosarcopenia: the randomized controlled FrOST study. Clin Interv Aging 2019;14:2173-86.

17 Kemmler W, Weineck M, Kohl M, et al. High intensity resistance exercise training to improve body composition and strength in older men with Osteosarcopenia. Results of the randomized controlled Franconian osteopenia and sarcopenia trial (frost). Front Sports Act Living 2020;2:1-12.

18 Kemmler W, Teschler M, Weißenfels A, et al. Prevalence of sarcopenia and sarcopenic obesity in older German men using recognized definitions: high accordance but low overlap! Osteoporos Int 2017:28:1881-91.

19 Kemmler W, von Stengel S, Schoene D. Longitudinal Changes in Muscle Mass and Function in Older Men at Increased Risk for Sarcopenia - The FrOST-Study. J Frailty Aging 2019;8:57-61.

20 Baumgartner RN, Koehler KM, Gallagher D, et al. Epidemiology of sarcopenia among the elderly in New Mexico. Am J Epidemiol 1998;147:755-63.

21 Cruz-Jentoft AJ, Baeyens JP, Bauer JM, et al. Sarcopenia: European consensus on definition and diagnosis: report of the European Working group on sarcopenia in older people. Age Ageing 2010;39:412-23.

$22 \mathrm{WHO}$. Assessment of osteoporotic fracture risk and its application to screening for postmenopausal osteoporosis. Geneva: World Health Organization, 1994.

23 DVO. Prophylaxe, Diagnostik und therapie der OSTEOPOROSE bei postmenopausalen Frauen und bei Männern Leitlinie des Dachverbands der Deutschsprachigen Wissenschaftlichen Osteologi schen_Gesellschaften_e.V. Schattauer: Stuttgart, 2017.

24 Steele J, Fisher J, Giessing J. Clarity in reporting terminology and definitions of set end points in resistance training. Muscle Nerve 2017;10:368-74.

25 Zourdos MC, Klemp A, Dolan C, et al. Novel resistance TrainingSpecific rating of perceived exertion scale measuring repetitions in reserve. J Strength Cond Res 2016;30:267-75.

26 Johnson JL, Slentz CA, Houmard JA, et al. Exercise training amount and intensity effects on metabolic syndrome (from studies of a targeted risk reduction intervention through defined exercise). Am J Cardiol 2007;100:1759-66.

27 Schoeffl I, Kemmler W, von Stengel S. Physical activity, strength and $\mathrm{VO}$ max have no significant influence on bone parameters in elderly women. J Musculoskelet Neuronal Interact 2008;8:363-74.

28 Honaker J, King G, Blackwell M. Amelia II: a program for missing data. JSS 2011;45:1-47.

29 Cohen J. Statistical power analysis for the behavioral sciences. 2nd edn. Hillsdale, NJ: Lawrence Earlbaum Associate, 1988: 8-16.

30 Donini LM, Busetto L, Bauer JM, et al. Critical appraisal of definitions and diagnostic criteria for sarcopenic obesity based on a systematic review. Clin Nutr 2020;39:2368-88.

31 Bauer JM, Cruz-Jentoft AJ, Fielding RA, et al. Is there enough evidence for Osteosarcopenic obesity as a distinct entity? A critical literature review. Calcif Tissue Int 2019;105:109-24. 
32 Bateman LA, Slentz CA, Willis LH, et al. Comparison of aerobic versus resistance exercise training effects on metabolic syndrome (from the Studies of a Targeted Risk Reduction Intervention Through Defined Exercise - STRRIDE-AT/RT). Am J Cardiol 2011;108:838-44.

33 Earnest CP, Johannsen NM, Swift DL, et al. Aerobic and strength training in concomitant metabolic syndrome and type 2 diabetes. Med Sci Sports Exerc 2014;46:1293-301.

34 Kemmler W, Wittke A, Bebenek M, et al. High intensity resistance training methods with and without protein supplementation to fight cardiometabolic risk in middle-aged males: a randomized controlled trial. Biomed Res Int 2016;2016:1-9.

35 Wittmann K, Sieber C, von Stengel S, et al. Impact of whole body electromyostimulation on cardiometabolic risk factors in older women with sarcopenic obesity: the randomized controlled FORMOsA-sarcopenic obesity study. Clin Interv Aging 2016;11:1697-706.

36 Denysschen CA, Burton HW, Horvath PJ, et al. Resistance training with soy vs whey protein supplements in hyperlipidemic males. J Int Soc Sports Nutr 2009;6:8.

37 Sheikholeslami Vatani D, Ahmadi Kani Golzar F. Changes in antioxidant status and cardiovascular risk factors of overweight young men after six weeks supplementation of whey protein isolate and resistance training. Appetite 2012;59:673-8.

38 Figueroa A, Okamoto T, Jaime SJ, et al. Impact of high- and low-intensity resistance training on arterial stiffness and blood pressure in adults across the lifespan: a review. Pflugers Arch 2019;471:467-78.

39 Liu C-J, Latham NK. Progressive resistance strength training for improving physical function in older adults. Cochrane Database Syst Rev 2009:CD002759.

40 Mangione KK, Miller AH, Naughton IV. Cochrane review: improving physical function and performance with progressive resistance strength training in older adults. Phys Ther 2010;90:1711-5.

41 Steele J, Fisher J, Skivington M, et al. A higher effort-based paradigm in physical activity and exercise for public health: making the case for a greater emphasis on resistance training. BMC Public Health 2017:17:300.

42 Winett RA, Ogletree AM. Evidence-based, high-intensity exercise and physical activity for compressing morbidity in older adults: a narrative review. Innov Aging 2019;3:igz020.
43 Weinheimer EM, Conley TB, Kobza VM, et al. Whey protein supplementation does not affect exercise training-induced changes in body composition and indices of metabolic syndrome in middle-aged overweight and obese adults. J Nutr 2012;142:1532-9.

44 Fernandes RR, Nabuco HCG, Sugihara Junior P, et al. Effect of protein intake beyond habitual intakes following resistance training on cardiometabolic risk disease parameters in pre-conditioned older women. Exp Gerontol 2018;110:9-14.

45 Campbell WW, Kim JE, Amankwaah AF, et al. Higher total protein intake and change in total protein intake affect body composition but not metabolic syndrome indexes in middle-aged overweight and obese adults who perform resistance and aerobic exercise for 36 weeks. J Nutr 2015;145:2076-83

46 Cyarto EV, Brown WJ, Marshall AL. Retention, adherence and compliance: important considerations for home- and group-based resistance training programs for older adults. J Sci Med Sport 2006;9:402-12.

47 Kalinkovich A, Livshits G. Sarcopenic obesity or obese sarcopenia: a cross talk between age-associated adipose tissue and skeletal muscle inflammation as a main mechanism of the pathogenesis. Ageing Res Rev 2017;35:200-21.

48 Calvani R, Joseph A-M, Adhihetty PJ, et al. Mitochondrial pathways in sarcopenia of aging and disuse muscle atrophy. Biol Chem 2013;394:393-414.

49 Sullivan-Gunn MJ, Lewandowski PA. Elevated hydrogen peroxide and decreased catalase and glutathione peroxidase protection are associated with aging sarcopenia. BMC Geriatr 2013;13:104.

50 Bowen TS, Schuler G, Adams V. Skeletal muscle wasting in cachexia and sarcopenia: molecular pathophysiology and impact of exercise training. J Cachexia Sarcopenia Muscle 2015;6:197-207.

51 Kemmler W, Weineck J, Kalender WA, et al. The effect of habitual physical activity, non-athletic exercise, muscle strength, and VO2max on bone mineral density is rather low in early postmenopausal osteopenic women. J Musculoskelet Neuronal Interact 2004;4:325-34.

52 McAuley E, Konopack JF, Motl RW, et al. Measuring disability and function in older women: psychometric properties of the late-life function and disability instrument. J Gerontol A Biol Sci Med Sci 2005;60:901-9. 